\title{
Thermal Degradation Studies of Polyurethane/POSS Nanohybrid Elastomers
}

J. P. Lewicki, K. Pielichowski, P.

TremblotDeLaCroix, B. Janowski, D. Todd, J. J. Liggat

March 8, 2010

Polymer Degradation and Stability 
This document was prepared as an account of work sponsored by an agency of the United States government. Neither the United States government nor Lawrence Livermore National Security, LLC, nor any of their employees makes any warranty, expressed or implied, or assumes any legal liability or responsibility for the accuracy, completeness, or usefulness of any information, apparatus, product, or process disclosed, or represents that its use would not infringe privately owned rights. Reference herein to any specific commercial product, process, or service by trade name, trademark, manufacturer, or otherwise does not necessarily constitute or imply its endorsement, recommendation, or favoring by the United States government or Lawrence Livermore National Security, LLC. The views and opinions of authors expressed herein do not necessarily state or reflect those of the United States government or Lawrence Livermore National Security, LLC, and shall not be used for advertising or product endorsement purposes. 
Elsevier Editorial System(tm) for Polymer Degradation and Stability Manuscript Draft

Manuscript Number: PDST-D-09-00525R2

Title: Thermal Degradation Studies of Polyurethane/POSS Nanohybrid Elastomers

Article Type: Research Paper

Keywords: polyurethane; POSS; thermal degradation; stabilisation

Corresponding Author: Dr James Pawel Lewicki, Ph.D

Corresponding Author's Institution: Lawrence Livermore National Laboratory

First Author: James P Lewicki, Ph.D

Order of Authors: James P Lewicki, Ph.D; Krzysztof Pielichowski; Pauline Tremblot De La Croix; Bartlomiej Janowski; Deborah Todd, Msci; John J Liggat

Abstract: Reported here is the synthesis of a series of Polyurethane/POSS nanohybrid elastomers, the characterisation of their thermal stability and degradation behaviour at elevated temperatures using a combination of Thermal Gravimetric Analysis (TGA) and Thermal Volatilisation Analysis (TVA). A series of PU elastomers systems have been formulated incorporating varying levels of 1,2-propanediolheptaisobutyl-POSS (PHIPOSS) as a chain extender unit, replacing butane diol. The bulk thermal stability of the nanohybrid systems has been characterised using TGA. Results indicate that covalent incorporation of POSS into the PU elastomer network increase the non-oxidative thermal stability of the systems. TVA analysis of the thermal degradation of the POSS/PU hybrid elastomers have demonstrated that the hybrid systems are indeed more thermally stable when compared to the unmodified PU matrix; evolving significantly reduced levels of volatile degradation products and exhibiting a $\sim 30^{\circ} \mathrm{C}$ increase in onset degradation temperature. Furthermore, characterisation of the distribution of degradation products from both unmodified and hybrid systems indicate that the inclusion of POSS in the PU network is directly influencing the degradation pathways of both the soft and hard block components of the elastomers: The POSS/PU hybrid systems show reduced levels of $\mathrm{CO}, \mathrm{CO} 2$, water and increased levels of THF as products of thermal degradation. 



\title{
Thermal degradation studies of polyurethane/POSS nanohybrid elastomers
}

James P. Lewicki ${ }^{{ }^{*}}$, Krzysztof Pielichowski ${ }^{2}$, Pauline Tremblot De La Croix ${ }^{1}$, Bartlomiej Janowski ${ }^{2}$ Deborah Todd ${ }^{1} \&$ John J. Liggat ${ }^{1}$

${ }^{1}$ WestChem, Department of Pure and Applied Chemistry, University of Strathclyde, 295 Cathedral Street, Glasgow G1 1XL, UK

${ }^{2}$ Department of Chemistry and Technology of Polymers, Cracow University of Technology, ul. Warszawska 24, 31-155 Krakow, Poland

AUTHOR E-MAIL ADDRESSES: James P Lewicki - lewicki1@1lnl.gov ; John J. Liggat j.j.liggat@strath.ac.uk

*CORRESPONDING AUTHOR FOOTNOTE. Ph: (925) 4231115 Fax: (44) 1415484822 email: lewicki1@1lnl.gov

\begin{abstract}
Reported here is the synthesis of a series of polyurethane/POSS nanohybrid elastomers, the characterisation of their thermal stability and degradation behaviour at elevated temperatures using a combination of thermogravimetric Analysis (TGA) and thermal volatilisation analysis (TVA). A series of PU elastomers systems have been formulated incorporating varying levels of 1,2-propanediol-heptaisobutyl-POSS (PHIPOSS) as a chain extender unit, replacing butane diol. The bulk thermal stability of the nanohybrid systems has been characterised using TGA. Results indicate that covalent incorporation of POSS into the PU elastomer network increases the nonoxidative thermal stability of the systems. TVA analysis of the thermal degradation of the POSS/PU hybrid elastomers have demonstrated that the hybrid systems are indeed more thermally stable when compared to the unmodified PU matrix; evolving significantly reduced levels of volatile degradation products and exhibiting a $\sim 30^{\circ} \mathrm{C}$ increase in onset degradation temperature. Furthermore, characterisation of the distribution of degradation products from both unmodified and hybrid systems indicate that the inclusion of POSS in the PU network is directly influencing the degradation pathways of both the soft and hard block components of the elastomers: The POSS/PU hybrid systems show reduced levels of $\mathrm{CO}, \mathrm{CO}_{2}$, water and increased levels of THF as products of thermal degradation.
\end{abstract}

KEYWORDS: polyurethane; POSS; thermal degradation 


\section{INTRODUCTION}

Polyhedral oligomeric silsesquioxanes (POSS) are an increasingly well known and well studied class of discrete, 3-dimensional polycyclic compounds. Condensed silsesquioxanes have the general formula $\left(\mathrm{RSiO}_{1.5}\right)_{2 \mathrm{n}}$, where $\mathrm{n}$ is an integer and $\mathrm{R}$ can be a large number of substituents including hydrogen, alkyl, alkenyl, phenyl, halogen and siloxy groups. Common structures of silsesquioxanes include random, ladder and cube or cage type structures. Octameric cage structures (cages with eight silicon atoms) have the general formula $\left(\mathrm{R}_{8} \mathrm{Si}_{8} \mathrm{O}_{12}\right)$ and are $\sim 1.2 \mathrm{~nm}$ in diameter. These pseudo-cubic POSS cages are one of the most commonly encountered and studied examples of silsesquioxanes $(1,2,3)$.

Cubic POSS molecules have received much attention in recent years; mainly due to the fact that they are discrete, well defined nano-scale form of silica which can be readily modified with a wide range of organic substituents. This uniformity, small size scale and ease with which POSS can be decorated with reactive organic functionalities makes them, in many respects, ideal candidates for use as nano-scale physical and chemical property modifiers in polymeric systems (4).

With particular reference to polyurethanes, there has been much activity in recent years directed towards the covalent incorporation of POSS into segmented polyurethane elastomers in order to modify the structure of segmented polyurethane networks; with the goal of improving the physical properties of the elastomer. $\mathrm{Fu}$ et. al.(5) reported the incorporation of diol functionalised POSS cages into a segmented PU network and demonstrated by the use of X-ray scattering methods that POSS formed microcrystalline domains within the PU system - which are likely to contribute to the effective hard-block content of the elastomer. In a similar study, Bliznyuk (6) showed that when incorporated into a segmented PU system, aminopropyl and hydroxy functionalised POSS form highly crystalline nano-domains within the elastomer which were enriched with inorganic silica. In a follow up study, Fu (7) demonstrated that such POSS-PU hybrid systems have significantly improved mechanical properties; as a result of the formation of nano-crystalline silica domains. Liu and co-workers (8) also reported the synthesis of POSS-PU nanohybrid systems by incorporating Isocyanate functionalised POSS cages directly into a segmented PU elastomer. At low levels of inclusion, significant improvements in mechanical properties were observed; the hybrid systems 
displayed increases in $\mathrm{T}_{\mathrm{g}}$ and increased storage moduli over that of the un-modified base PU elastomer.

In general, work in recent years have shown that the introduction of comparatively low mass fractions of isocyanate or diol functionalised POSS effectively contributes to the hard block content of the elastomer system, reinforcing the mechanical properties of the elastomer as a whole. While this is of significance and interest, the focus of the work reported here is on the thermal degradative stability of such PU-POSS hybrid systems. There have been a number of studies published which report on the thermal properties of segmented PU-POSS hybrid systems: Liu (8) reported that PUPOSS hybrid elastomers had enhanced thermal stability over that of base PU elastomers from a series of TGA experiments. Liu also speculated that the primary mechanism of PU degradation was not significantly altered and the enhanced stability was due to a condensed-phase action of POSS in the degrading polymer melt. Madbouly et. al (9) presented similar results from TGA studies of POSS-PU hybrids; their results suggested that inclusion of POSS into a PU network does not significantly effect the onset of non-oxidative thermal degradation, but does enhance the high temperature thermal stability - leading to higher char yields and a decrease in rate of late-stage, high temperature degradation. Similar degradation behaviour was also reported in the work of Zhang and co-workers (10); where diol-functionalised POSS incorporated into a PU network did not alter the onset of thermal degradation, but increased the final char yield. Indeed, in a recent study by the authors (11), the thermo(oxidative stability) of POSS/PU nanohybrid systems were investigated. It was shown that these nanohybrid materials had increased thermal stability and maximum rate degradation temperatures that were shifted to higher temperatures, when compared to unmodified PU systems. The results were explained in terms of a restriction in the global mobility of the PU chains in the presence of POSS - leading to a reduction in the rate of degradation reactions and volatiles evolution.

While such studies are important in characterising the thermal properties of novel POSS-PU hybrid systems, TGA studies in isolation offer little insight into the mechanisms of the degradation of the PU matrix. Segmented PU elastomers are inherently complex systems; consisting of regions of elastomeric polyol 'soft-block' linked by rigid crystalline, isocyanate 'hard-block' domains. The 
range of polyols, isocyanates, chain extenders and other modifiers that can be used in the synthesis of PU elastomers is extensive. The cure chemistry of these systems is also complex, with urea-linkage reactions often competing with the formation of urethane bonds. As such, there is often a range of many different chemical functionalities in any given PU and it is difficult to define any single PU structure as 'typical' (12). Despite this complexity, mechanistic studies of high temperature PU degradation have demonstrated a general commonality in the mechanism of degradation in PU elastomers: Grassie et. al $(13,14)$ carried out mechanistic TVA degradation studies on a polyether polyurethane formed from butane diol and Methylene bis-(4-phenyl diisocyanate) (MDI). The results of this work demonstrated that although there were a wide variety of products of degradation formed (including: carbon monoxide, $\mathrm{CO}_{2}$ butadiene, THF, dihydrofuran, water and $\mathrm{HCN}$ ), the overall degradation reaction can be explained in terms of a simple depolymerisation process (which occurs in the region of $200^{\circ} \mathrm{C}$ ) whereby the isocyanate and diol monomers are reformed. The complex mixture of products observed is subsequently formed from the monomers in the melt phase at temperatures above $300^{\circ} \mathrm{C}$ as a result a series of secondary condensation, elimination and radical scission reactions. This behaviour is at least superficially consistent with what is reported in the literature for the degradation of POSS-PU hybrid systems: The fact that the onset of primary thermal degradation appears in general, to be unaffected by the inclusion of POSS into the matrix suggests that the primary de-polymerization of urethane linkages is unaffected by the inclusion of low levels of POSS as a component of the matrix. However, increased char yields and shifts in the rate of high temperature degradation in the melt point towards the fact that POSS may be influencing the secondary stage degradation of the re-generated monomers. In order to better understand the mechanistic changes occurring in the degrading PU matrix - the composition and distribution of the degradation products must be investigated in detail.

In this work we report characterisation of the degradation behaviour and (products thereof) of a series of POSS-PU hybrid systems by combined use of TGA and TVA. The use of TVA has allowed the mechanisms of primary and secondary degradation to be studied as a function of the relative quantities of volatile products evolved during degradative thermal analysis.

\section{EXPERIMENTAL}


2.1 Materials. The polyurethane matrix was prepared by reaction of 4,4'methylenebis(phenylisocyanate) (MDI) as isocyanate component and poly(tetramethylene glycol) (TERATHANE $1400{ }^{\circledR}$ ) with molecular weight of $\sim 1400$ as the elastic component. 1,4-butanediol was used as chain extender. Nanocomposites were prepared by appropriate substitution of chain extender by 1,2-propanediol-heptaisobutyl-POSS (PHIPOSS) in order to provide samples with POSS mass fractions of $2-10 \%$. The mass fraction of elastic component in the polyurethane was $50 \%$.

2.2 Preparation polyurethane/POSS nanohybrid elastomers. For the purposes of this study, a series of six POSS-PU hybrid elastomer systems were prepared incorporating $0,2,4,6,8$ (designated P1 to P6 respectively) and 10\% PHIPOSS as a substitute chain extender. The elastomer systems were prepared in the following manner: MDI was charged into a $100 \mathrm{ml}$ three-necked round bottomed flask, equipped with a mechanical stirrer and nitrogen inlet. The MDI was heated to $70^{\circ} \mathrm{C}$ and a solution of PHIPOSS in suitable amount of Terathane polyol was then added in one portion. (The PHIPOSS/polyol mixture had been previously prepared by heating the mixture to $120^{\circ} \mathrm{C}$ to dissolve the POSS in the polyol and then cooling to $60^{\circ} \mathrm{C}$ ). The polymerization reaction was performed under a nitrogen atmosphere at $80^{\circ} \mathrm{C}$ for $2 \mathrm{~h}$ to form a polyurethane prepolymer. The NCO group content was then determined and the prepolymer was mixed with suitable amount of 1,4butanediol. The resultant mixture was poured out on Petri dish, cured at $110^{\circ} \mathrm{C}$ for $2 \mathrm{~h}$ and finally post-cured at $80^{\circ} \mathrm{C}$ in $16 \mathrm{~h}$ to form a solid elastomer.

2.3 Thermogravimetric Analysis. A Netzsch thermogravimetric analyzer TG 209 was used to investigate the thermal stability of the obtained elastomers. $\sim 4 \mathrm{mg}$ samples were heated in an open $\alpha$ $\mathrm{Al}_{2} \mathrm{O}_{3}$ pan, from $25^{\circ} \mathrm{C}$ up to $600^{\circ} \mathrm{C}$ at a heating rate of $10^{\circ} \mathrm{C} / \mathrm{min}$ under an argon atmosphere. Onset degradation temperatures were defined for individual thermograms as the point at which a $2 \%$ mass loss occurred during the analysis.

2.2 Thermal Volatilization Analysis. All TVA analysis was carried out using a TVA line which was built in-house, based upon the apparatus and techniques described by McNeill et al. (15) The apparatus consisted of a sample chamber (heated by a programmable tube furnace) connected in series to a primary liquid nitrogen cooled sub-ambient trap and a set of four secondary liquid nitrogen cooled cold traps. The whole system was continuously pumped to a vacuum of $1 \times 10^{-4}$ torr 
by means of a two stage rotary pump and oil diffusion pumping system. Volatile condensable products could be initially trapped at two stages: The water jacket cooled 'cold ring' $\left(\mathrm{T} \sim 12{ }^{\circ} \mathrm{C}\right)$ immediately above the heated area of sample tube (this condensed high boiling point materials) and the primary liquid nitrogen cooled sub-ambient trap $\left(\mathrm{T} \sim-196^{\circ} \mathrm{C}\right)$ which collected all the lower boiling point species. Two linear response Pirani gauges were positioned at the entrance and exit of the primary sub-ambient trap to monitor the evolution of both condensable and non-condensable volatiles as a function of pressure vs. temperature/time from the sample. The use of linear response Pirani gauges allows valid pressure peak integrations to be carried out; where peak area corresponds to the quantity of evolved volatiles. Trapped, low-boiling species could be distilled into separate secondary cold traps by slowly heating the primary sub-ambient trap to ambient temperatures. These separated fractions could be subsequently removed into gas-phase cells for FTIR and GC-MS analysis. A series of non-linear Pirani gauges were placed at the entrance and exits of all secondary fraction traps to monitor the pressure changes as volatile species were distilled into separate traps and gas cells.

All TVA runs were conducted under vacuum using $25 \mathrm{mg}$ samples of each model system. The heating rate was $20^{\circ} \mathrm{C} \min ^{-1}$ to a temperature $550^{\circ} \mathrm{C}$. A 1-300 amu Hiden single quadrupole RGA mass spectrometer sampled a continuous product stream during both the degradation and differential distillation runs. Sub-ambient differential distillation of collected volatiles was carried out by heating the primary sub-ambient trap at a rate of $4^{\circ} \mathrm{C} \min ^{-1}$ from -196 to $40^{\circ} \mathrm{C}$. Volatiles were separated into four major fractions for subsequent IR and GC-MS analysis. A significant cold-ring fraction was also collected for each sample.

All FTIR analysis of the collected TVA products was carried out using a Mattson 5000 FTIR Spectrometer used in transmission mode. High boiling 'cold ring' fractions were cast from chloroform solution onto $\mathrm{NaCl}$ disks for analysis. Low-boiling volatiles were analyzed in the gas phase using gas phase cells with $\mathrm{NaCl}$ windows.

All GC-MS analysis of the collected TVA products was carried out using a Finnigan ThermoQuest capillary column trace GC and Finnigan Polaris Quadrapole Mass Spectrometer. Suitable fractions were dissolved in chloroform and subsequently analyzed. 


\section{RESULTS AND DISCUSSION}

3.1 TGA of POSS-PU hybrid elastomer systems. Samples of each elastomer system modified with 0-10\% of PHIPOSS were analysed using TGA under a non-oxidative (argon) atmosphere in order to characterise the thermal stability of each system. In addition to the six elastomer systems, a sample of PHIPOSS was also analysed using TGA. The TGA plots showing percentage mass loss as a function of temperature are given for all systems in Figure 1

From Figure 1 it can be observed that under non-oxidative conditions there appears to be a small, yet significant increase in the onset degradation temperature for the majority of the POSS-PU hybrids when compared with the unmodified (base) elastomer: At loadings of 2, 6, 8 and $10 \%$ there is an observed increase in onset degradation temperature of $\sim 10^{\circ} \mathrm{C}$ from 284 to $295^{\circ} \mathrm{C}$. The $4 \%$ POSS-PU elastomer does not follow this trend and appears to show a mass loss at significantly reduced temperatures. It is also apparent from the TGA data in Figure 1 that both the maximum rate of degradation and the final char yields are affected by the introduction of POSS into the PU elastomer systems: With increasing POSS loading, the rate of mass loss increases at temperatures above $300^{\circ} \mathrm{C}$ and the total mass loss after $600^{\circ} \mathrm{C}$ increases. The TGA data would certainly seem to suggest that the POSS-PU hybrid systems have modified thermal degradation behaviour. The increase in onsetdegradation temperature suggests that the POSS-PU hybrids are more thermally stable with respect to the primary de-polymerisation, whist the increased rate of mass loss and lower char yields may be indicative of an acceleration of secondary stage degradation processes in the hybrid systems.

\subsection{Degradative TVA analysis of POSS-PU hybrid elastomer systems. Degradative TVA was} carried out on all PU elastomer systems. The degradation was followed as a function of pressure of evolved volatile degradation products vs. temperature and time. The thermal degradation of the PU systems under high vacuum in each case produced a significant quantity of condensable, noncondensable volatile species, and a semi-volatile 'cold-ring' fraction. The TVA plots showing the rate of total volatiles evolution vs. temperature for each PU system are presented in Figure 2. There are four main stages (numbered 1-4) in the TVA plots obtained from the degradation of the PU systems. These stages have been assigned as: 1 - Loss of water from the sample at low temperatures 
and other low molecular weight reaction residues, 2 - escape of small inclusions of entrained air upon melting, 3 - onset of the primary degradation associated with the de-polymerization of urethane bonds and 4 - the high temperature radical scission stage associated with the secondary degradation of the regenerated polyol, resulting in the main release of non-condensable degradation products.

From Figure 2 it can clearly be observed that the un-modified PU systems evolve the greatest relative quantity of volatile degradation products and that the onset of main-stage volatiles evolution (associated with the onset of primary thermal degradation) occurs at significantly increased temperatures in the POSS-PU hybrid systems. The onset degradation temperatures; defined as point at which system pressure reaches $1 \times 10^{-3}$ torr during the heating ramp have been calculated for each system and are presented in Table 1. From Table 1 it can be observed that the onset degradation temperature of the un-modified PU system is $\sim 260^{\circ} \mathrm{C}$. The POSS-PU hybrid systems in contrast have onset degradation temperatures ranging from $\sim 285$ to $295^{\circ} \mathrm{C}$. This clearly indicates that the POSSPU hybrid systems are in fact significantly more thermally stable that the unmodified PU elastomer. The $2 \%$ POSS-PU system was the most thermally stable having and onset-degradation temperature of $294 \pm 2{ }^{\circ} \mathrm{C}$ and the $8 \%$ system was comparatively, the least stable having an onset-degradation temperature of $287 \pm 2{ }^{\circ} \mathrm{C}$. These data therefore show a similar trend to the TGA results, however the absolute values differ somewhat due to instrumental factors.

As previously stated, all of the systems studied evolved small, yet significant quantities of noncondensable volatile gasses. The release of these products occurred at temperatures in excess of $300^{\circ} \mathrm{C}$ and they are associated with the secondary degradation stage. This is illustrated in Figure 3 which compares the condensable and non-condensable traces from the degradation of the unmodified PU system. From Figure 3 it can be observed that the non-condensable gas is a small yet significant component of the total volatiles produced during degradation. On-line gas-phase MS carried out during each run has identified the gas as Carbon Monoxide in all cases. CO is a typical degradation product of PU systems associated with the high temperature radical scission of the polyol. Shown in Figure 4 are the composite TVA plots of the non-condensable gas evolved for each sample. It can be observed that there is a reduction in $\mathrm{CO}$ evolution in the hybrid systems. In order to compare the relative quantities of total and non-condensable products evolved from each sample, the 
peak areas from Figure 4 have been calculated. Table 2 summarises the relative quantities of volatile gases evolved from each sample as a function of relative peak area. It is clear from Table 2 that all of the POSS-PU hybrid systems evolve significantly reduced levels of volatile degradation products when compared to the base PU elastomer. The 2 and 4\% systems show the greatest reduction, typically evolving $\sim 50 \%$ less volatiles. This pronounced reduction is indicative of a major change in the degradation behaviour of the polyurethane system.

Overall, the results of degradative TVA demonstrate that the POSS-PU hybrids are significantly more thermally stable than the base PU system and that the ratio of volatile to non-volatile degradation products over the range of $20-550^{\circ} \mathrm{C}$ has shifted significantly in the hybrid systems. The reduction in volatiles suggests that a greater proportion of the degradation products formed over this temperature range are of increased boiling point and hence molar mass. The formation of larger involatile chain fragments combined with a reduction in the rate of diffusion of low molecular weight volatile species through the hybrid systems may account for the observed reduction in overall volatiles evolution.

\subsection{Differential distillation and characterisation of collected degradation products. The} collected volatile products for all the PU systems studied were separated by sub-ambient distillation after each degradation run. The differential distillation plots for each system are shown in Figure 5. Individual peaks represent discrete components of the total volume of collected volatile species. The individual major volatile products of degradation have been identified, primarily through gas-phase MS and correspond to peaks labelled 1-7 on Figure 5. The products have been identified as: $1-$ Formaldehyde, $2-\mathrm{CO}_{2}, 3-$ Butene + trace propanal, $4-\mathrm{THF}, 5-$ Toluene (trace), $6-$ Water and 7 - a mixture of 4 and 5 carbon glycol residues. A trace of ethane was also detected and is not shown due to scale. All of these compounds are products of the thermal degradation of the polyol, the chain extender (butane diol) and MDI. CO, Formaldehyde, propanal and the higher fragments are all thought to be products of high temperature radical scission of the polyol (Butene is a related elimination product). A general scheme for the radical scission of the polyol chain is given in Figure 6. THF is likely to form from the dehydration of butane diol. A proportion of the water observed will have originated from physically entrained water within the sample. Indeed, at moderate temperatures 
during each degradation run $\left(100-150^{\circ} \mathrm{C}\right)$ condensable volatile gasses were evolved at low levels. This is consistent with 'out-gassing' of small quantities of water, free diol or solvent from the samples as they are heated under vacuum. The levels of water observed in the sub-ambient distillation of products are however not consistent with out-gassing alone. A large proportion of the water observed is therefore attributed to the degradation of the PU systems. Water can be formed at high temperatures from the condensation of butane diol and similar condensation reactions of higher segments of the polyol. The general reaction pathways for the formation of these products are outlined in Figure 7. Toluene is thought to be a degradation product of MDI within the system. A general scheme for its formation is outlined in Figure 7 (scheme B). In line with existing model studies of MDI degradation (16), it is thought that a small yet significant level of MDI undergoes degradation with the elimination of $\mathrm{CO}_{2}$ to form an aromatic amine. Nucleophilic attack at the methyl bridge will yield a free aromatic amine which, at high temperatures $\left(>400^{\circ} \mathrm{C}\right)$ will decompose further, yielding toluene and ammonia. Indeed, a repeat TVA degradation of the unmodified PU system carried out up to a temperature of $350^{\circ} \mathrm{C}$ confirmed that initial degradation peak assigned as the primary depolymerisation stage (stage 3 on Figure 2) consisted of a mixture of $\mathrm{CO}_{2}$, butene, THF and water. Toluene, $\mathrm{CO}$, formaldehyde, propanal and the higher glycol residues were all absent at this stage of the degradation and it can therefore be concluded that they are formed as secondary degradation products at temperatures in excess of $400^{\circ} \mathrm{C}$ (stage 4 on Figure 2).

The cold-ring fraction was also analysed for each degradation and found to be very similar for each system. Shown in Figure 8 is the FTIR spectrum of the collected cold-ring fraction, obtained from the degradation of the base PU elastomer. From FTIR analysis of the cold-ring in Figure 8, the main functional groups present have been identified. The assignments are given in Table 3 . The bound $\mathrm{OH}$, aliphatic $-\mathrm{CH}$ and $\mathrm{C}-\mathrm{O}$ stretches indicate that a significant proportion of the cold-ring fraction consists of the polyol or fragments thereof. The other main component would appear to be MDI this is evident from the strong isocyanate peak at $2277 \mathrm{~cm}^{-1}$, the aromatic contributions at $\sim 3500 \mathrm{~cm}^{-1}$ and in the fingerprint region. The presence of free MDI in the cold-ring fraction was also confirmed by GC-MS. There is also some evidence from the FTIR analysis of the presence of species 
bearing cumulated double bonds - most likely due a carbodiimide. Much like aromatic amines, carbodiimides are secondary degradation products of the thermal decomposition of aromatic isocyanates such as MDI (13). It is therefore clear that a proportion of the MDI has thermally degraded within the polymer systems. However, from a simple qualitative assessment of the levels of free MDI in the cold-ring and the low levels of secondary degradation products observed, it seems apparent that the majority of the MDI is lost from the system 'unscathed' during the initial depolymerisation stage of the degradation process.

It is clear from the characterisation of the volatile degradation products, that the PU systems are following the general degradation pathways outlined in existing studies $(13,14)$ and the products formed can be categorised as either decomposition products of the polyol or isocyanate components of the PU systems. The introduction of POSS into the PU network is not inducing the formation of significant levels of previously unreported degradation products; however it is clear from Figure 5, that the relative quantities of volatile products of degradation are altered significantly in the hybrid systems. $\mathrm{CO}_{2}-\mathrm{a}$ product of decomposition of the isocyanate within the polyurethane matrix is reduced significantly in all hybrid systems: The 2 and $4 \%$ systems evolve the smallest relative quantity of $\mathrm{CO}_{2} \cdot \mathrm{CO}_{2}$ levels then increase somewhat though the 6,8 and $10 \%$ systems. Butene levels remain effectively constant in all systems - suggesting that its formation is independent of the presence of POSS. THF is somewhat interesting as it again remains generally constant in all systems with the notable exception of the $6 \%$ POSS-PU hybrid which exhibited a $\sim 65 \%$ increase in THF production over the base PU. This increase would immediately suggest that there is a greater quantity of butane diol available to form THF and may be an indication that the $6 \%$ sample is anomalous in that respect. The 2, 4, 6 and $8 \%$ hybrids all show a significant reduction in water evolution when compared with the PU system - an indication of a reduction in dehydration reactions. The $10 \%$ sample exhibits a large increase in water when compared to even the base system - again this is difficult to interoperate unless this sample was significantly different in its composition to all other systems.

In general, the profile of degradation products demonstrates that the reduction in volatiles is primarily due to an associated decrease in the evolution of $\mathrm{CO}, \mathrm{CO}_{2}$ and water from these systems as 
they degrade. The reduction in $\mathrm{CO}$ evolution clearly points towards a reduction in radical chain scission in the POSS-PU hybrid systems and the decrease in $\mathrm{CO}_{2}$ evolution suggests that MDI decomposition is altered by the introduction of POSS. The reduction in water evolution effectively indicates that there is a reduction in the number of alcohol de-hydrations. If these observations are applied to what is known about PU degradation, then it becomes clear that the covalent inclusion of POSS is influencing the high temperature degradation of the polyol and isocyanate segments of the PU systems in addition to stabilising it towards primary de-polymerization. The overall result of this action is to increase the thermal stability of the PU matrix. It is thought that the nano-scale silica particles, present as chain extender segments within the PU matrix are effectively increasing the level of hard-block domains within the polyurethane. Indeed, in a related study by the authors it has been shown that these POSS-modified systems have increased $T_{g}$ values over the unmodified elastomer (17). POSS therefore physically re-enforces the PU matrix. These higher $\mathrm{T}_{\mathrm{g}}$ systems exhibit increased thermal stability and show a reduction in volatile degradation products above $300^{\circ} \mathrm{C}$. This behaviour, although in some respects being at odds with a number of the PU systems reviewed in the introduction is consistent with the Vyazovkin model (18), which attributes the observed enhancement in the thermal stability in PU/POSS hybrid systems in terms to a reduction in the molecular mobility of the polymer chains by the bulky oligosilsesquioxane pendent groups and the increased level of crystalline domains. With reduced molecular mobility, the diffusion of reactive species will be curtailed to an extent throughout the system and the diffusion of volatile degradtion products out of the system with also be reduced (19). These materials would therefore be expected to have reduced reactivity, greater thermal stability and a reduced yield of volatile degradation products.

\section{CONCLUSIONS}

This study has demonstrated that the covalent inclusion of POSS nano-silica particles into a PU matrix increases the thermal stability of such systems - resulting in an increase in degradation onset temperature and a significant reduction in the overall level of volatile degradation products formed; 
which in turn points to a reduction in radical chain scission, secondary MDI decomposition and urethane bond depolymerisation. POSS physically re-enforces the PU systems and is thought to reduce the rate both of reactive species transport and of degradation product diffusion within the PU matrix - effectively stabilising the POSS/PU hybrids towards thermal decomposition.

\section{ACKNOWLEDGEMENTS}

This work has been partially supported by the Polish Ministry of Science and Higher Education under contract No. N N507 365733.

This work performed under the auspices of the U.S. Department of Energy by Lawrence Livermore National Laboratory under Contract DE-AC52-07NA27344. 


\section{REFERENCES}

1. Feher FJ, Budzichowski TA, Weller KJ. Journal of the American Chemical Society 1989;111: 7288 .

2. Mason BW, Morrison JJ, Coupar PI, Jaffres P, Morris RE. Journal of the Chemical Society, Dalton Transactions 2001:1123.

3. Janowski B, Pielichowski J. Advances in Polymer Science 2006;201:225.

4. Joshi M, Butola BS. Journal of Macromolecular Science, Part C - Polymer Reviews 2004;44:389.

5. Fu BX, Hsiao BS, White H, Rafailovich M, Mather PT, Jeon HG, Phillips S, Lichtenhan JS, Schwab J. Polymer International 2000;49:437.

6. Bliznyuk VN, Tereshchenko TA, Gumenna MA, Gomza YP, Shevchuk AV, Klimenko NS, Shevchenko VV. Polymer 2008;49:2298.

7. Fu BX, Hsiao BS, Pagola S, Stephens P, White H, Rafailovich M, Sokolov J, Mather PT, Jeon HG, Phillips S, Lichtenhan J, Schwab J. Polymer 2001;42:599.

8. Liu H, Zheng S. Macromolecular Rapid Communications 2005;26:196.

9. Madbouly SA, Otaigbe JU, Nanda AK, Wicks DA. Macromolecules 2007;40:4982

10. Zhang S, Zou O, Wu L. Macromolecular Materials and Engineering 2006;291:895.

11. Janowski B, Pielichowski K. Thermochimica Acta 2008;478:51.

12. Woods G. “The ICI Polyurethanes book” Second edition 1990 J Wiley and Sons.

13. Grassie N, Zulfiquar M. Journal of Polymer Science Part A - Polymer Chemistry 1978;16:1563. 
14. Grassie N, Scott G. "Polymer Degradation and Stabilisation" 1985, Cambridge University Press, Cambridge, pp. 39-41.

15. McNeill IC, Ackerman L, Gupta SN, Zulfiquar M, Zulfiquar S. Journal of Polymer Science Part A: Polymer Chemistry 1977;15:2381.

16. Lewandowski G, Milchert E. Journal of Hazardous Materials 2005;1:19.

17. Raftopoulos K, Pandis C, Apekis L, Pissis P, Pielichowski K, Janowski B. "Modern polymeric Materials for Environmental Applications” 2008, Vol. 3, Ed. K. Pielichowski, pp. 209-214.

18. Vyazovkin S, Dranca I, Fan X, Advincula R. Journal of Physical Chemistry B 2004;108:11672.

19. Liu H, Zheng H. Macromolecular Rapid Communications 2005;26:196.

\section{FIGURE CAPTIONS}

Figure 1. Composite TGA mass loss plots for 0 to $10 \%$ POSS-PU hybrid systems. P1 to P6 correspond to $0,2,4,6,8 \& 10 \%$ POSS loadings respectively.

Figure 2. Composite degradative TVA traces for 0 to $10 \%$ POSS-PU hybrid systems. P1 to P6 correspond to $0,2,4,6,8 \& 10 \%$ POSS loadings respectively.

Figure 3. Comparative degradative TVA trace showing both condensable and non-condensable volatile species evolved from the base PU elastomer system. Solid line denotes total volatile evolution and dashed line, the non-condensable volatile component.

Figure 4. Composite degradative TVA traces for 0 to $10 \%$ POSS-PU hybrid systems showing the non-condensable gas fraction of each system. Solid, dashed, dotted lines, squares, circles and triangles correspond to the $0,2,4,6,8 \& 10 \%$ POSS filled systems respectively. 
Figure 5. Composite sub-ambient differential distillation traces for 0 to $10 \%$ POSS-PU hybrid systems showing the distribution and relative quantities of volatile degradation products as a function of pressure vs. trap temperature. P1 to P6 correspond to $0,2,4,6,8 \& 10 \%$ POSS loadings respectively

Figure 6 . General reaction scheme showing the mechanisms and likely products of high temperature polyol radical chain scission.

Figure 7. General reaction scheme showing a) dehydration of butane diol to form THF and b) A probable mechanism for MDI degradation.

Figure 8. Transmittance FT-IR spectrum of the recovered cold-ring from the unmodified P1 system, having a strong isocyanate peak at $2277 \mathrm{~cm}^{-1}$.

\section{TABLE CAPTIONS}

Table 1. Onset degradation temperatures as defined during degradative TVA for all systems

Table 2. Relative quantities of non-condensable and total volatiles evolved during each TVA run for

all systems studied. Increased POSS levels correspond to a decrease in the levels of volatile degradation products observed.

Table 3. Peak assignments for Figure 8, the FT-IR Spectrum of the cold-ring fraction. For each peak, the position, strength/shape and a functional group assignment are given. 
Table 1

P1 (Base PU)

P2 (2\% POSS)

P3 (4\% POSS)

P4 (4\% POSS)

P5 (4\% POSS)

P6 (4\% POSS)
$262 \pm 2^{\circ} \mathrm{C}$

$294 \pm 2^{\circ} \mathrm{C}$

$293 \pm 2{ }^{\circ} \mathrm{C}$

$291 \pm 2^{\circ} \mathrm{C}$

$287 \pm 2^{\circ} \mathrm{C}$

$290 \pm 2^{\circ} \mathrm{C}$ 
Sample

P1 (Base PU)

P2 (2\% POSS)

P3 (4\% POSS)

P4 (6\% POSS)

P5 (8\% POSS)

P6 (10\% POSS)
Total volatiles evolved / torr $\mathbf{s}$

$$
\begin{aligned}
& 1.63 \pm 0.01 \\
& 0.80 \pm 0.01 \\
& 0.80 \pm 0.01 \\
& 1.17 \pm 0.01 \\
& 1.01 \pm 0.01 \\
& 1.01 \pm 0.01
\end{aligned}
$$

Non-condensable volatiles /torr $\mathbf{s}$

$$
\begin{aligned}
& 0.19 \pm 0.01 \\
& 0.05 \pm 0.01 \\
& 0.05 \pm 0.01 \\
& 0.03 \pm 0.01 \\
& 0.03 \pm 0.01 \\
& 0.05 \pm 0.01
\end{aligned}
$$




$\begin{array}{lll}\begin{array}{l}\text { Wavenumber } / \mathbf{c m}-1 \\ 3309\end{array} & \begin{array}{l}\text { Strength/shape } \\ \text { Strong, Rel. narrow }\end{array} & \begin{array}{l}\text { Assignment } \\ \text { bound } \mathrm{OH} \text { stretch }\end{array} \\ \sim 3100 & \text { Weak } & \text { Ar. C-H stretch } \\ 2939 \& 2856 & \text { Strong, narrow } & \mathrm{CH}_{2} \text { and } \mathrm{CH}_{3} \text { stretching } \\ 2796 & \text { shoulder } & \mathrm{O}-\mathrm{CH}_{2}-\mathrm{O} \\ 2277 & \text { Strong, narrow } & \text { Isocyanate } \\ 2150 \& 2100 & \text { weak doublet } & \text { carbodiimide } \\ 1750 \& 1700 & \text { doublet } & \text { Carbonyl groups } \\ 1111 & \text { Strong } & \text { C-O Stretch }\end{array}$


Click here to download high resolution image

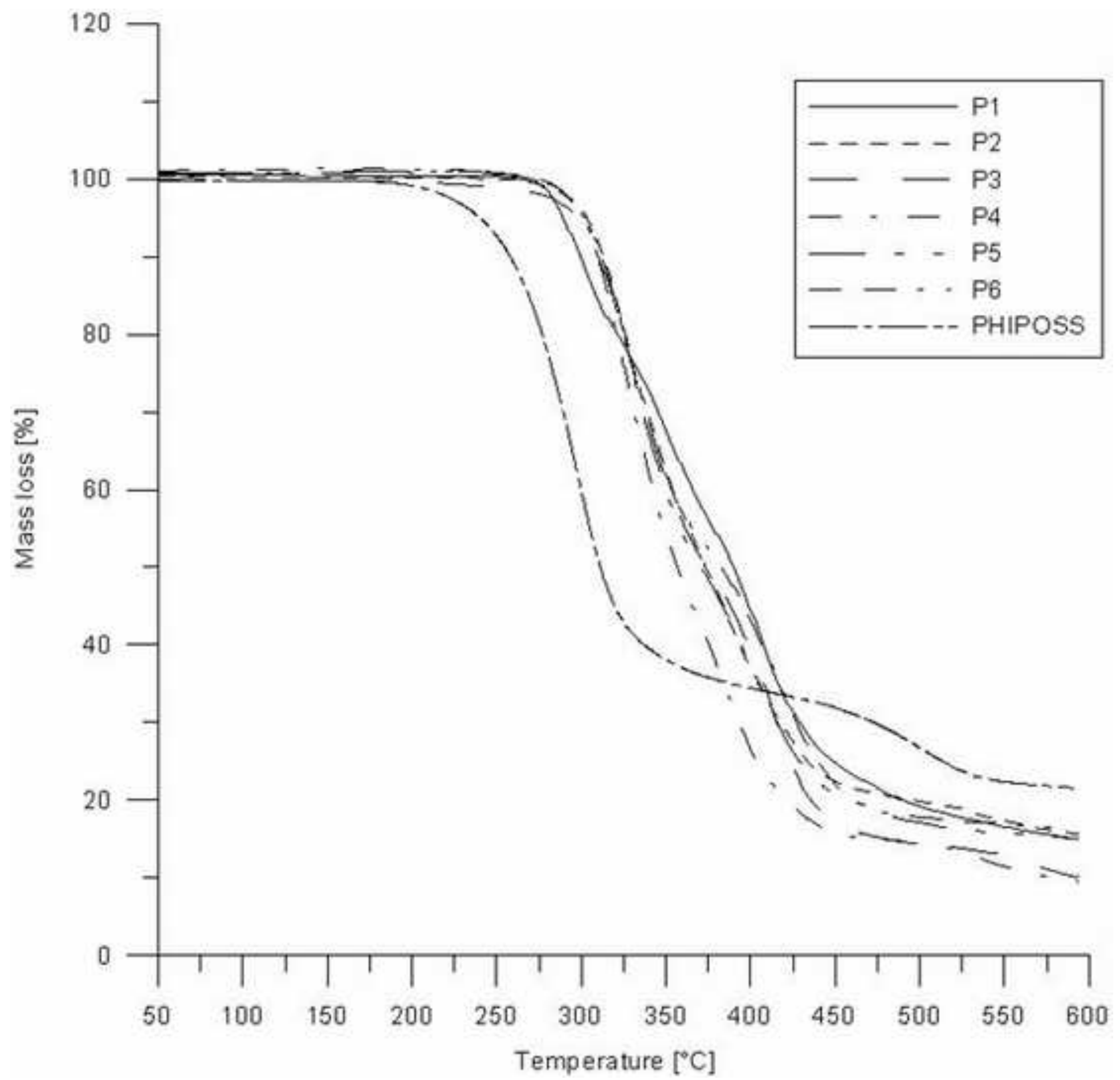




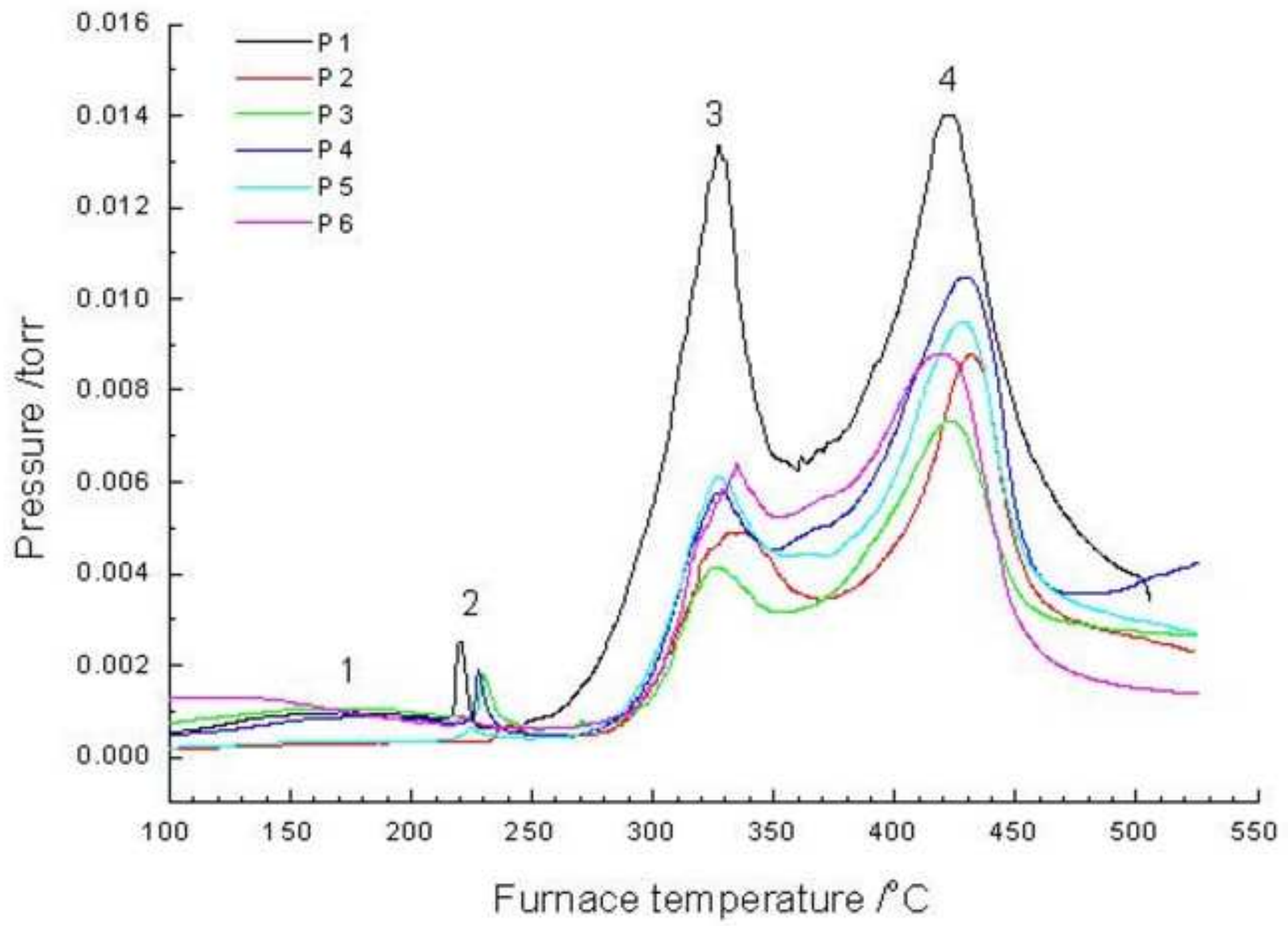




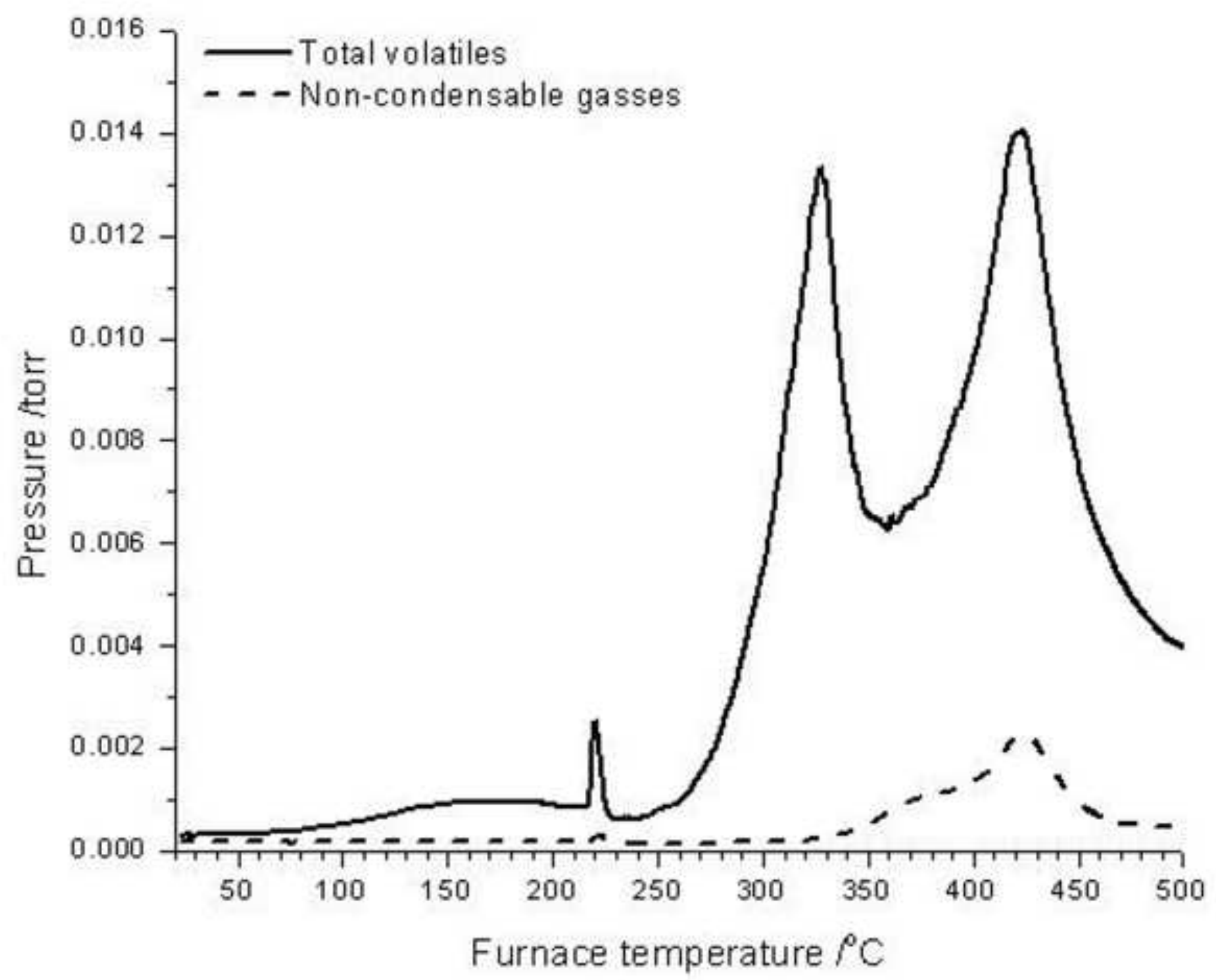




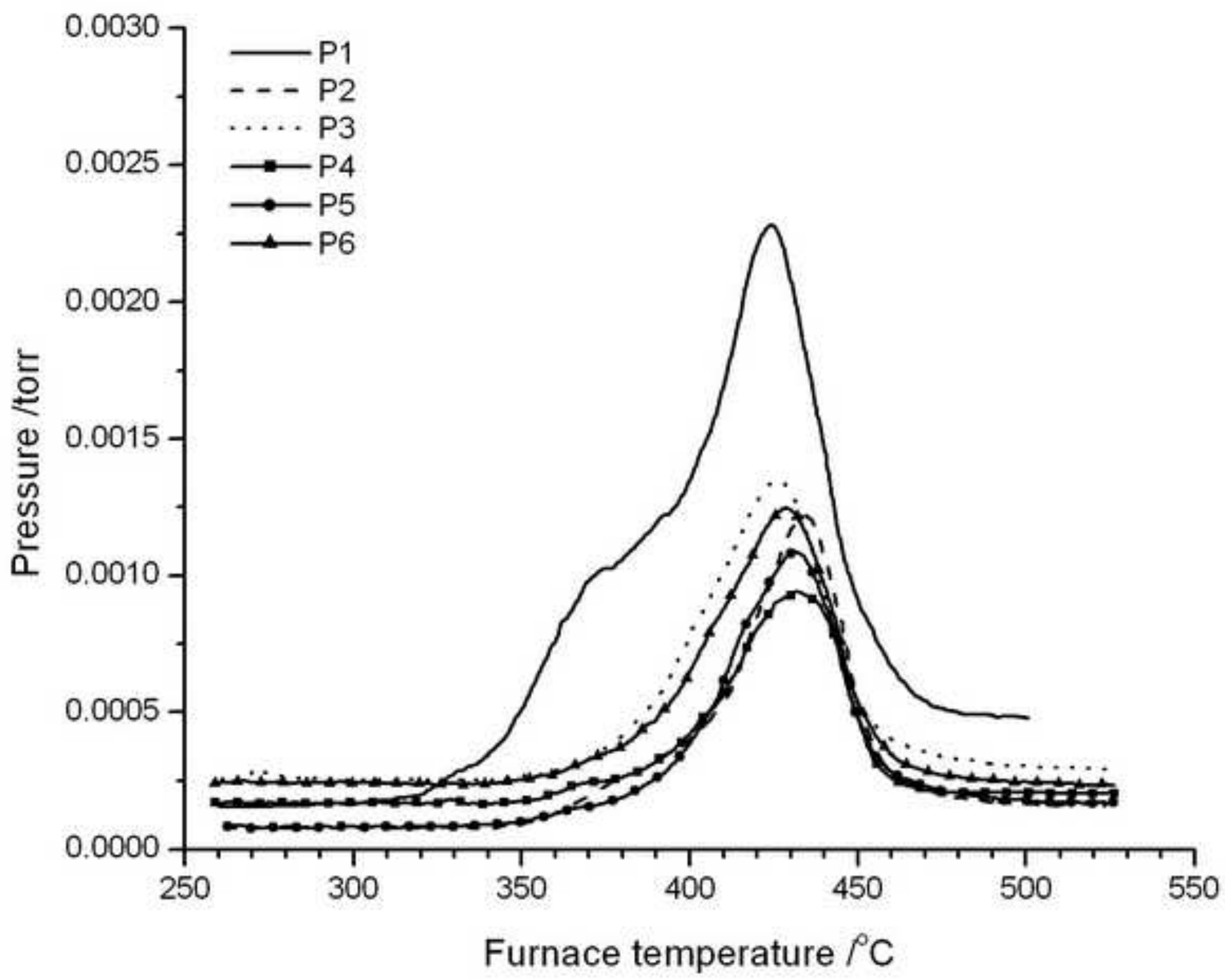




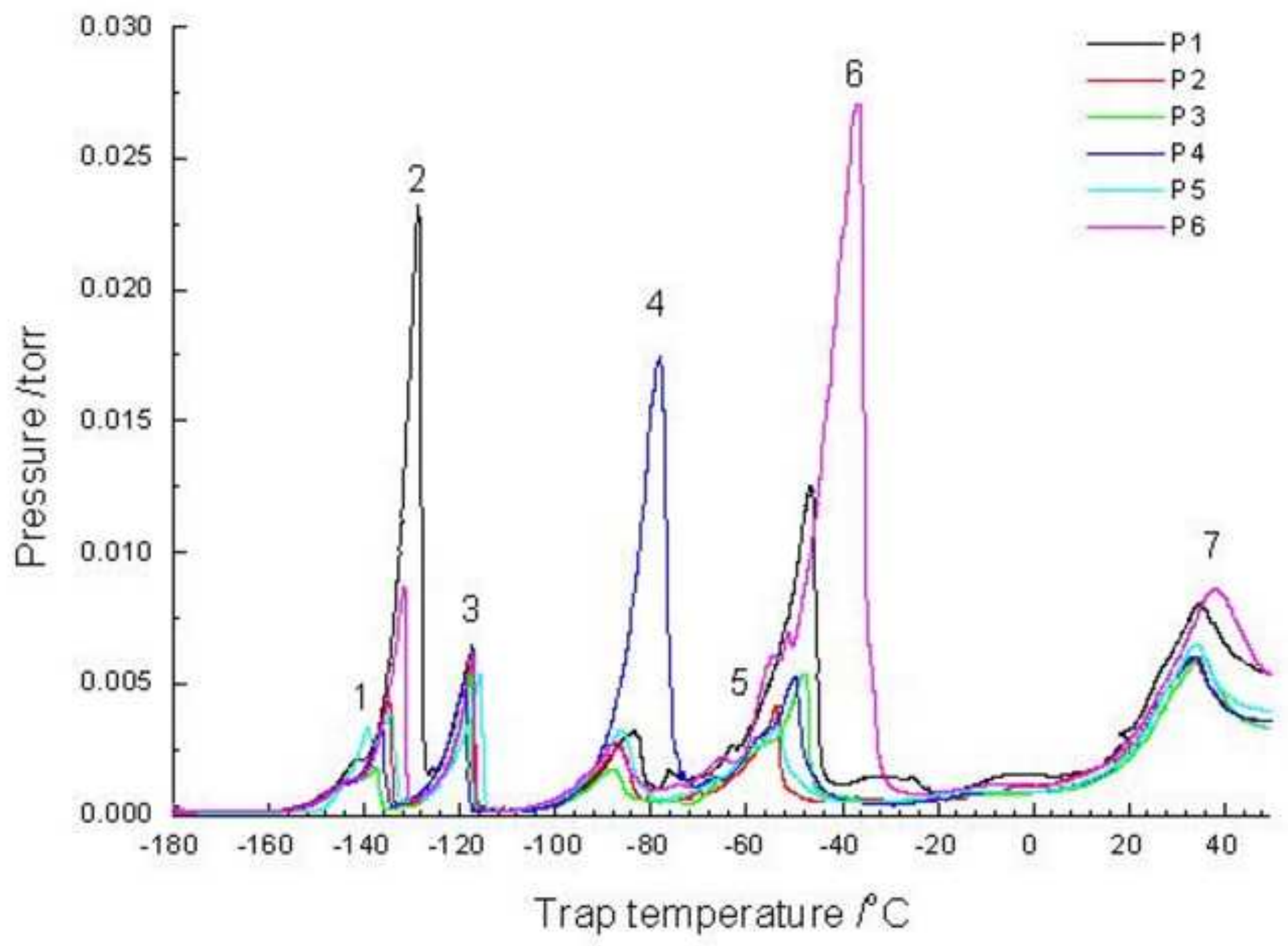


Click here to download high resolution image<smiles>COCCCCOCCCCOC</smiles>

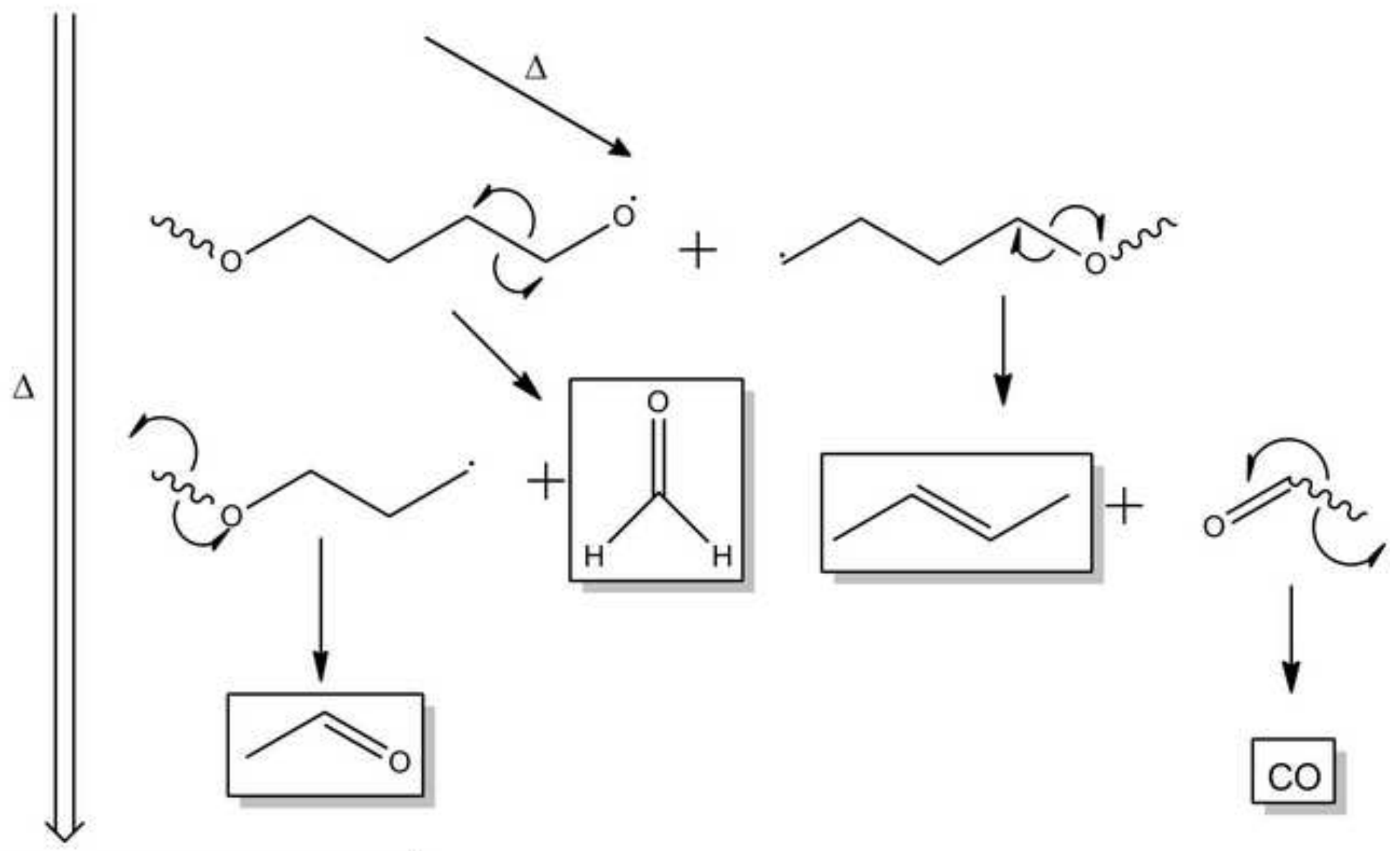

\section{Random chain scission \\ over two or more glycol residues \\ will form larger chain fragments}




\section{Figure 7}

Click here to download high resolution image
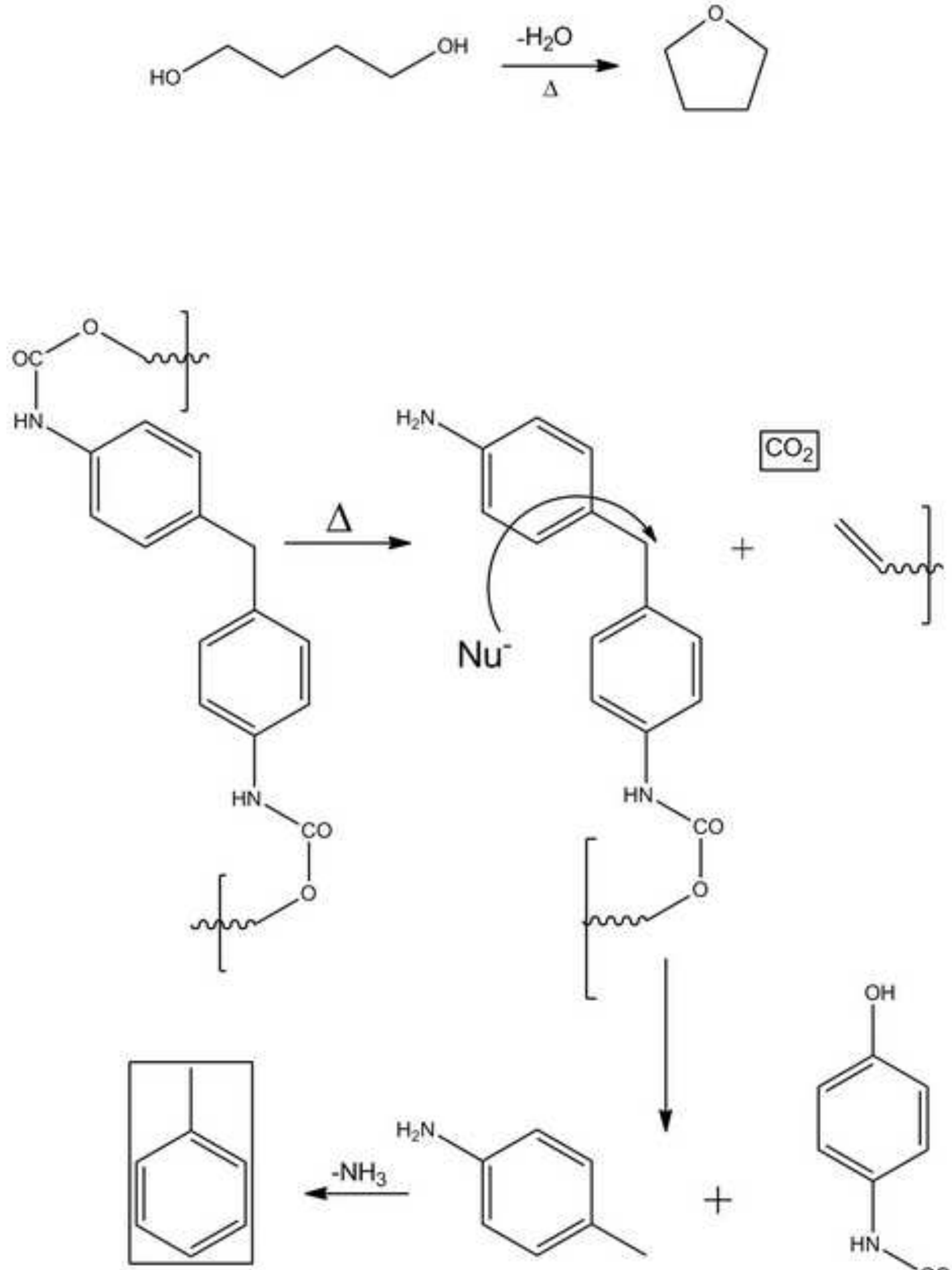

$+$

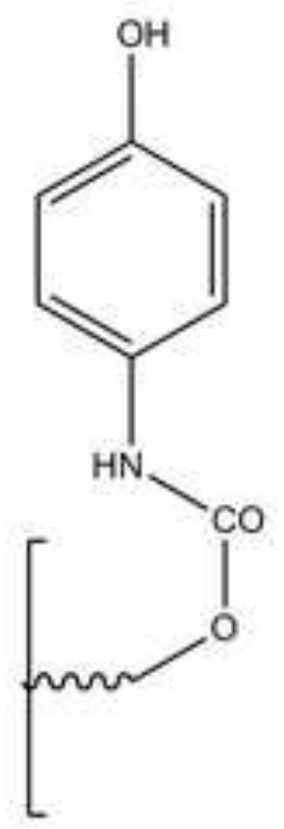




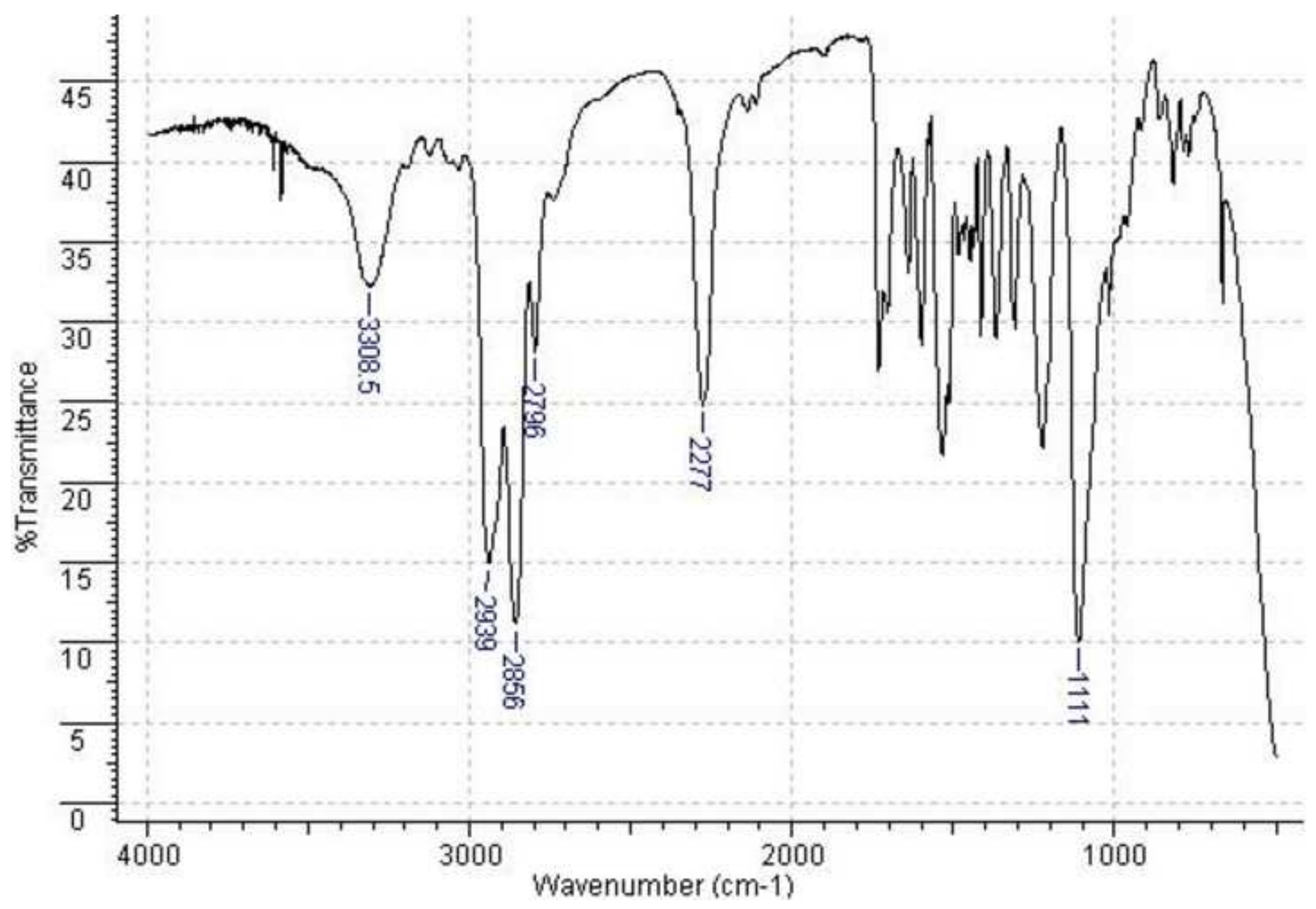


Shown below in Figure 1 is a TVA plot of the degradation under vacuum of a $25 \mathrm{mg}$ sample of Acupol® polyether polyol (MW $\sim 6000 \mathrm{~g} \mathrm{~mol}^{-1}$ ). This polyol consists of polypropylene glycol chains tipped with polyethylene glycol. When this polyol degrades, a percentage of the volatile products evolved are non-condensable gasses (red trace, Figure 1). This gas mixture was identified by online mass spectrometry as carbon monoxide, methane and low levels of hydrogen.

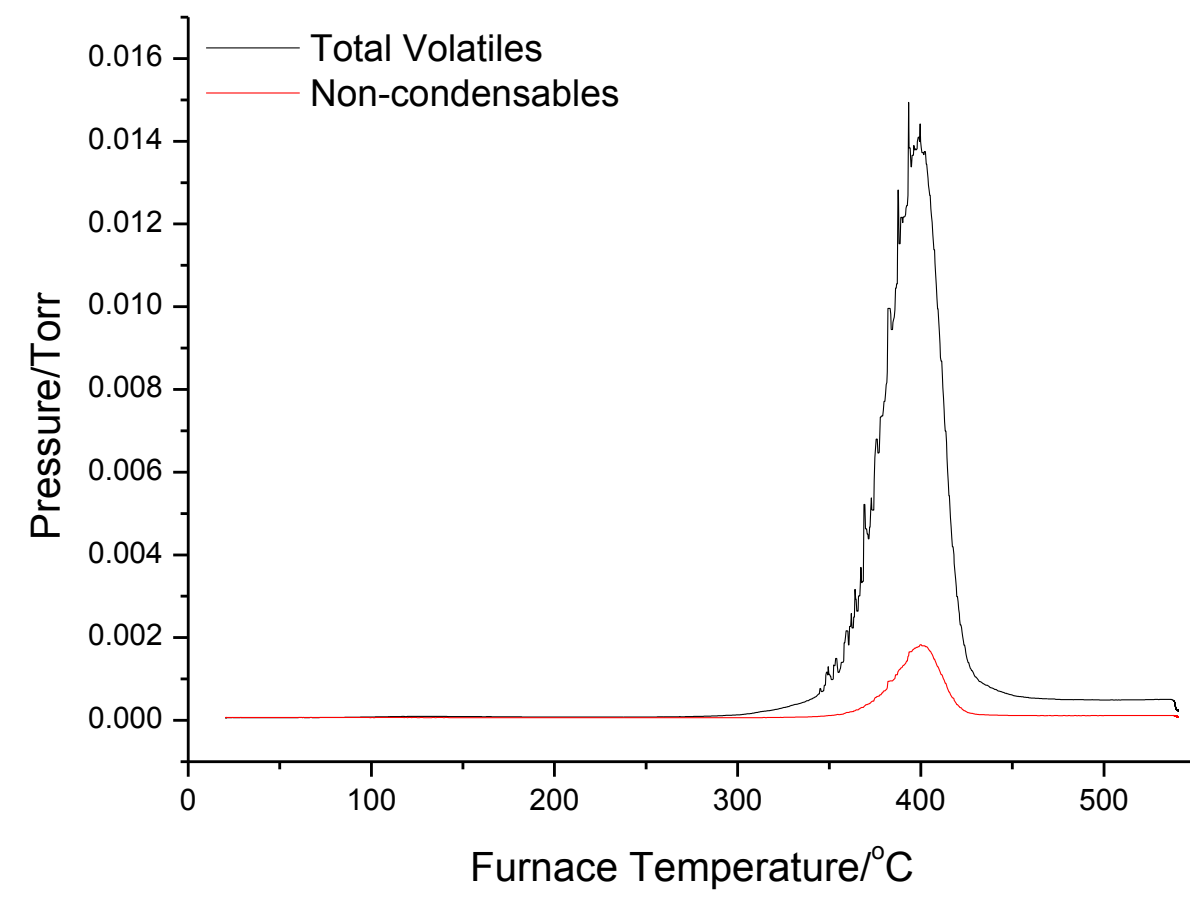

Figure 1. TVA degradation plot of a $25 \mathrm{mg}$ sample of an Acupol Polyether Polyol

Shown in Figure 2 is a Multiple Ion Detection (MID-MS) plot of the evolved noncondensable gases showing the identity and relative quantities in partial pressure of each consistent of the gas mixture 


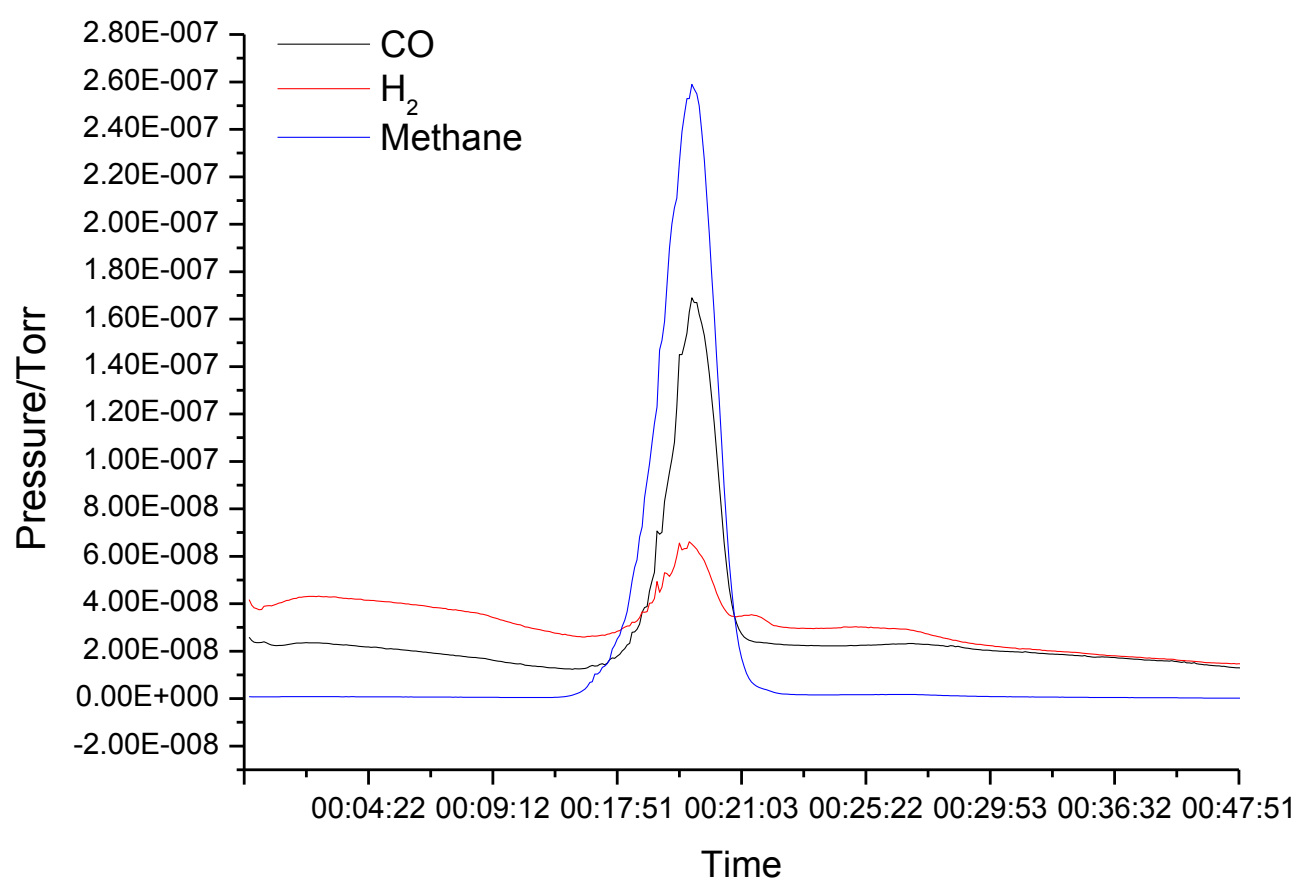

Figure 2. MID-MS plot of the non-condensable gas fraction of the polyol degradation showing the relative quanities of the 3 gasseous consitiuents present in this fraction

From the experimental data presented here it is evident that that polyether polyols do in fact evolve $C O$, methane and hydrogen as degradation products; as stated in the original manuscript. (In contrast to the poly(tetramethylene glycol) discussed in the main manuscript, this polyol evolves significant levels of methane, whereas in the PTMG system only $C O$ and hydrogen are observed). This experimental data is supported by numerous examples in the literature, which have been cited in the accompanying response to the reviewers. 